\title{
Complexity studies and human organizations
}

Complexity studies/theory has been emerging as a critical domain that provides better explanation and understanding of numerous human associated disciplines (economics, social, environmental, political, education, military) involving human activities, organizational structures and dynamics, leadership and management, self-organization/self-transcending constructs, and emergence that cannot be easily comprehend with the Newtonian mindset. All human organizations/systems are complex adaptive systems. A new mindset and paradigmatic shift appears to be inevitable when dealing (structuring, leading, managing, strategizing) with any systems/organizations comprising human interacting agents. The complexity and nonlinearity of human thinking systems (intelligence, consciousness, knowledge intensity) coupled with the rapid changing external environment introduce rising complexity into all human organizations.

This special issue on Complexity Studies and Human Organizations encompasses a fairly wide spread of research areas and researchers. It began with a brief recapture on the historical development of complexity studies by me. I also introduced the sub-domain of relativistic complexity in human organizations, and conceptualized the edge of emergence. My paper also provided an analysis of the new intelligence leadership strategy and relativistic complexity in human organizations. In the next article, Bill (US/France), Meng (China), Haoyue (China), and Richard (Australia) introduced and analyzed the NKF model (a modification of Kauffman's NK model) covering groupthink, strongtie and weak-tie in human organizations with respect to epistasis, the fitness landscape, and complexity catastrophe. Their findings include biological epistasis equates with strong-tie connection; and all results of NK modeling show movement towards complexity catastrophe as the value of $\mathrm{K}$ increases.

The third paper by David (Singapore) examined leadership learning through the lens of complexity theory and Popper's three worlds. The study involved more than 300 participants in a development program (Leaders in Education Program) for potential school principals at the National Institute of Education in Singapore. The fourth paper by Desmond (US) proposed a theoretical model for entrepreneurs that operate in a context of ambiguity under uncertain settings characterized by complexity. The analysis covered several perspectives including complexity as a source of ambiguity, ambiguity aversion and overconfidence, entrepreneurial overconfidence as a response to ambiguity aversion, and complexity and 'illusion of control' bias. The study observed that overconfidence is a response to ambiguity aversion, and the latter reflects rewards to an entrepreneur's judgments for seeing opportunities through a fog of ambiguity. Finally, the last paper by Michelle (Australia), Abel (Australia) and Harry (Australia) examined the complexity posed by contemporary changes, competition, globalization, and technology, and the way their developments have impacted the professional Australian Rugby Union organization. The overall findings indicate the significance of the four characteristics in the organization studied.

For leaders and managers in any human organizations/communities/groups to venture into complexity a new complexity mindset and understanding is essential. Currently, most of them do not possess a clear/holistic feel of how complexity theory or concepts/knowledge on complex adaptive systems can be explored and exploited. The recognition of the co-existence of 
order and complexity in human organizations, and that leading and managing such systems requires an integrated deliberate and emergent approach is a vital starting point. Next, realizing that the primary goal of complexity studies focuses on the emergent of order (an intrinsic characteristic of complex adaptive systems) is also a crucial necessity. Subsequent analyses, exploration, and exploitation at a more micro level will have to be supported by these two basic characteristics.

Thow Yick Liang Guest Editor 